\title{
OBSERVATIONS OF THE OUTER ATMOSPHERIC REGIONS OF $\alpha$ ORIONIS
}

\author{
A.P. Bernat and L. Goldberg \\ Kitt Peak National Observatory* \\ Tucson, AZ 85726 , U.S.A.
}

Abstract

We present three separate observational studies of mass flows above the photosphere of $\alpha$ ori (M2 Ia-Ib). The Ca II infrared triplet lines and Ha are asymmetric showing a systematic blue shift with decreasing residual intensity. These lines remain fixed in wavelength although the weak photospheric lines vary by $\pm 4 \mathrm{~km} / \mathrm{sec}$. Observations of the $4.6 \mu$ vibration-rotation spectrum of CO show two sharp, cold components expanding at velocities of 10 and $17 \mathrm{~km} / \mathrm{sec}$ relative to the centre of mass. Direct photographs of the shell in the light of $\mathrm{KI} \lambda 7699$ show that the cold shell is asymetxic and extends outward to at least $50 "$ ".

Details of these studies are either in press or will be submitted to "The Astrophysical Journal".

* Operated by the Association of Universities for Research, Inc. under contract with the National science Foundation. 